\title{
Evaluación de riesgos, monitorización y simulación de edificios patrimoniales
}

\author{
Carmen M.a Muñoz González, Ángel Luis León Rodríguez, Jaime Navarro Casas, \\ Jonathan Ruiz Jaramillo, Catherine Teeling
}

\begin{abstract}
Resumen: Los edificios religiosos son una parte importante del patrimonio cultural, son documentos de nuestra herencia y tenemos la necesidad de preservarlos. Las condiciones ambientales en el que están inmersos estos edificios son determinantes para la preservación y conservación del patrimonio mueble que contiene. Originalmente, la mayoría de estos espacios no estaban acondicionados, pero actualmente, estos edificios están siendo climatizados para proporcionar el confort térmico a los feligreses y para mejorar las condiciones del clima interior y la preservación del patrimonio mueble. El objetivo principal de esta investigación ha sido analizar las condiciones ambientales de edificios religiosos en un clima Mediterráneo, mediante el uso de monitorización y simulación para evaluar y mejorar la preservación del patrimonio cultural de estos edificios. Este estudio aporta las herramientas para la optimización de la calidad ambiental para la preservación y conservación del patrimonio mueble e inmueble y para su aplicación en futuros proyectos de rehabilitación patrimonial.
\end{abstract}

Palabras clave: Patrimonio, monitorización, simulación, riesgo, condiciones ambientales

\section{Evaluación de riesgos, monitorización y simulación de edificios patrimoniales}

Abstract: The churches are an important part of Cultural Heritage; these need to be preserved. The indoor environmental conditions of these spaces are decisive for the preservation and conservation of the movable heritage The originally churches were not conditioned, but currently, these buildings are being air-conditioned to provide thermal comfort to parishioners and to improve indoor weather conditions. The main objective of this research project has been to analyse the environmental conditions of religious buildings in a Mediterranean climate, through the use of monitoring and simulation to evaluate and improve the preservation of the Cultural Heritage of these buildings. Finally, this study provides the subject expert, the tools for the optimization of environmental and energy quality, in the refurbishment project.

Keywords: Heritage, monitoring, simulation, risk, environmental conditions

\section{Introducción}

El estudio higrotérmico de espacios religiosos protobarrocos se enmarca dentro una fase de estudios de investigación que tiene por objeto la futura rehabilitación de dichos espacios, y se pretende sirva de base para conocer el estado de conservación, los fenómenos de degradación que sufren algunas obras de arte y analizar previamente las consecuencias de las posibles intervenciones en los edificios.

Los procesos de degradación son resultado de la interacción de diversos parámetros ambientales tanto del exterior como el interior (Muñoz-Gonzalez 2016).
Uno de los objetivos de este estudio fue aportar datos que pudieran dar explicación a la degradación actual de los espacios religiosos analizados, debido a las condiciones ambientales interiores, a los factores climatológicos, el uso actual, etc. La observación de los parámetros ambientales interiores (obtenidos con una previa monitorización del espacio de estudio) desde un punto de vista de la dinámica ambiental, permite conocer el funcionamiento ambiental del espacio interior (Erhardt \& Mecklenburg 1994), (Magrini 2016). Debido a la naturaleza oscilante del clima, el proceso de monitorización se debe realizar para un periodo de varios años lo que permite un conocimiento más detallado de la interacción de los materiales y obras 
de arte con el clima interior (Wessberga \& Vyhlídal). Igualmente, debido a la influencia de la masa humana en la degradación y el confort térmico del espacio, este estudio ahonda en el conocimiento más detallado del uso de estos espacios y la interacción de las personas con el ambiente interior (Camuffo et al. 2010).

Un elevado porcentaje de edificios patrimoniales son contenedores de una riqueza inestimable de nuestro patrimonio cultural. Estos espacios albergan un patrimonio mueble, tan importante como el que se puede encontrar en espacios expositivos, los museos. En este ámbito poco a poco se han ido imponiendo diferentes estrategias de conservación, al menos como planteamiento teórico. Sin embargo, otros espacios como por ejemplo, los religiosos, son instituciones que no disponen de estructuras técnicas y administrativas permanentes para la conservación de los bienes que acopian, a pesar de que en la mayoría de los casos, muchos de ellos están inscritos en el Registro General de Bienes de Interés Cultural o forman parte del Inventario General de Bienes Muebles (DIRECCIÓN GENERAL DE BELLAS ARTES Y BIENES CULTURALES Y DE ARCHIVO $Y$ BIBLIOTECA 2009).

Además de las carencias en medios técnicos, es preciso considerar otra particularidad que puede determinar de forma importante la conservación del patrimonio, que es su carácter de patrimonio en uso. Generalmente, los espacios de culto están vinculados a ritos y tradiciones con un importante seguimiento popular en el que enlazan, además, elementos del patrimonio inmaterial que requieren asimismo una aproximación especial en cuanto a los requerimientos de conservación.

En los últimos treinta años, las iglesias están disminuyendo drásticamente, sobre todo en el occidente de Europa. Asimismo, muchos de estos espacios han tenido que ser usados para fines alternativos con el fin de disponer de algunos ingresos extras. El uso original de las iglesias está siendo modificado y actualmente a estos espacios, hoy por hoy, no se les exigen un acondicionamiento ambiental, como al resto de otras edificaciones que albergan actividades de uso público. Hoy en día muchas de ellas se utilizan para conciertos y otros eventos musicales, exposiciones, etc. (Mosoarca et al. 2017)

Durante siglos el clima interior de las iglesias había venido determinado principalmente por el clima exterior. Sin embargo, en la actualidad muchos de estos espacios están siendo equipados con novedosas tecnologías (diseñadas para una arquitectura contemporánea) para mejorar las condiciones de confort de los feligreses (Berardinis, Rotilio \& Capannolo 2017).

Igualmente, si el funcionamiento de estas técnicas ambientales fuese continuo, existiría una alta demanda de energía debido al gran volumen e inercia térmica que presentan estos edificios. Precisamente por razones económicas la mayoría de las iglesias emplean un sistema de climatización para períodos de tiempo limitados, concretamente cuando las personas están presentes. Pero esta estrategia de climatización puede tener un efecto adverso sobre la conservación del edificio y sus objetos interiores (Balocco \& Colaianni 2018).

Los problemas a los que se enfrenta la conservación de estos espacios están relacionados con la evaluación de riesgos, la aplicación de control y el seguimiento de las condiciones ambientales, una vez implementados los sistemas de acondicionamiento ambiental. En la mayoría de los casos, la implantación de las instalaciones de acondicionamiento ambiental deriva de dos necesidades principales, una es la económica (de la instalación, uso y mantenimiento) y la segunda es el confort térmico. Sin embargo, los requisitos de conservación en pocas ocasiones son considerados (Muñoz 2016).

Por esto, resulta relevante el presente trabajo, donde se propone profundizar en el conocimiento del acondicionamiento higrotérmico de espacios religiosos. Se pretende con ello, ofrecer a los técnicos competentes las pautas de diseño que le permitan predecir la conducta higrotérmica de estos espacios antes y después de la aplicación de técnicas ambientales.

\section{Metodología}

Este trabajo de investigación se desarrolló siguiendo un método experimental, depurado durante años de trabajo por parte de miembros del grupo de investigación TEP 130, adscrito al Instituto Universitario de Arquitectura y ciencias de la Construcción (IUACC) de la Universidad de Sevilla. Este método combina las formulaciones analíticas, las medidas experimentales realizadas in situ y la utilización de técnicas de simulación mediante ordenador para conocer y predecir el comportamiento higrotérmico de los espacios.

El método desarrollado para el análisis de cada iglesia objeto de estudio puede ser desglosado en cinco fases, las cuales se suceden cronológicamente.

- En la primera etapa del estudio se realiza una recopilación de información básica de la muestra de estudio. La documentación histórica permite conocer las circunstancias que propiciaron la construcción del edificio, su propia evolución y del entorno hasta nuestros días.

- Una segunda etapa, el seguimiento del ambiente interior cuya finalidad es proporcionar datos objetivos de parámetros higrotérmicos para realizar la evaluación y diagnosis del estado de conservación el patrimonio cultural y el confort térmico. Las iglesias fueron monitorizadas durante veintidós meses, excepto la iglesia de nuestra señora de la Victoria, que por mantenimiento 
1. Toma de datos y recopilación de información básica

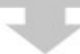

2. Monitorización y realización de mediciones in situ, procesado y valoración higrotérmica

3. Elaboración de modelos tridimensionales para la simulación del campo higrotérmico y ajuste de los modelos informáticos a partir de las mediciones in situ

4. Propuestas de mejoras del acondicionamiento ambiental de la muestra para la conservación y el confort térmico y simulación informática de las propuestas.

5. Análisis y valoración de las condiciones ambientales tras la implantación de técnicas ambientales

Figura 1.- Fotografía de emplazamiento y levantamiento planimétrico.

y obras solo se pudo hacer un seguimiento ambiental durante dieciséis meses.

- En la tercera etapa se elaboran los modelos tridimensionales para la simulación del campo higrotérmico. Los datos de la monitorización se emplean para generar los modelos de simulación informática, elaborando modelos digitales tridimensionales de cada caso de estudio. Estos modelos espaciales reproducen cada edificio y su entorno, desde el punto de vista geométrico, climático y constructivo, empleando para ello el programa informático Design Builder.

Este modelo informático se valida a partir de las mediciones in situ. La metodología de validación se basa en la comparación de un importante número de datos horarios registrados in situ con los resultados obtenidos de la simulación informática una vez generado el modelo.

- Una cuarta etapa donde se desarrollan propuestas de mejoras del acondicionamiento ambiental de la muestra mediante la aplicación de técnicas ambientales pasivas y activas en los modelos de simulación, para la conservación y el confort térmico.

- Finalmente, en la quinta etapa se analiza y valora los resultados obtenidos de los modelos informáticos para determinar la adecuación de las condiciones ambientales para la conservación, confort térmico y eficiencia energética.

El esquema propuesto para la gestión de las condiciones ambientales en ningún caso tiene como objetivo proporcionar soluciones específicas o reglas sobre cómo conseguir estándares de niveles estrictos de dichas condiciones, sino que pretende proporcionar una metodología de trabajo normalizados que permite tomar decisiones adecuadas y coordinadas sobre futuras intervenciones en edificios históricos, sin poner en riesgo el bien patrimonial.

\section{Caso de estudio}

Para este estudio se analizaron tres espacios religiosos que se encontraban en un mismo ámbito geográfico y por lo tanto tenían unas condiciones climatológicas externas muy similares. Los espacios religiosos se encontraban en la localidad de Morón de la Frontera, en la provincia de Sevilla (España). Estas iglesias, forman parte del patrimonio inmueble de Andalucía, algunas de las mismas declaradas BIC (Bien de Interés Cultural), con la categoría de monumento, como es el caso de la Iglesia de San Francisco de Asís

Las iglesias, pertenecían a un mismo periodo constructivo, comprendido entre 1550-1650 para poder realizar un estudio comparativo. Estas presentaban variaciones en cuanto a forma de planta, sistema constructivo de cubierta, huecos de ventanas, etc., factor que enriquecía el estudio, ya que se podía determinar la influencia de estas características sobre el comportamiento higrotérmico en las mismas.

En la figura 2, se muestran las tres iglesias de estudio, la primera, la iglesia de San Francisco de Asís del año 1550, presenta una planta en forma cajón, cubierta por una bóveda de cañón y lunetos reforzados por pares de arcos fajones. La planta está orientada de manera que los muros longitudinales, se encuentran, con una ligera desviación al norte. En los alrededores de la iglesia están adosados las demás dependencias como capillas, casa parroquial y sobre una de las medianeras se levanta el claustro del antiguo monasterio. La segunda, la iglesia Nuestras Señora de la Merced del 1638, de planta cruz latina con una falsa bóveda de cañón con arcos fajones y lunetos que cubren los brazos del crucero, mientras que una bóveda semiesférica sobre pechinas cubre el crucero. Por último, la iglesia Nuestra Señora de la Victoria del año 1584, formada por una nave de cinco tramos, con capillas unidas mediante arcos de medio punto. La nave se cubre con tejado a dos aguas cuyo interior está cubierto con un artesonado de madera. A continuación, se muestra las características constructivas de las iglesias analizadas, véase [tabla 1]. 


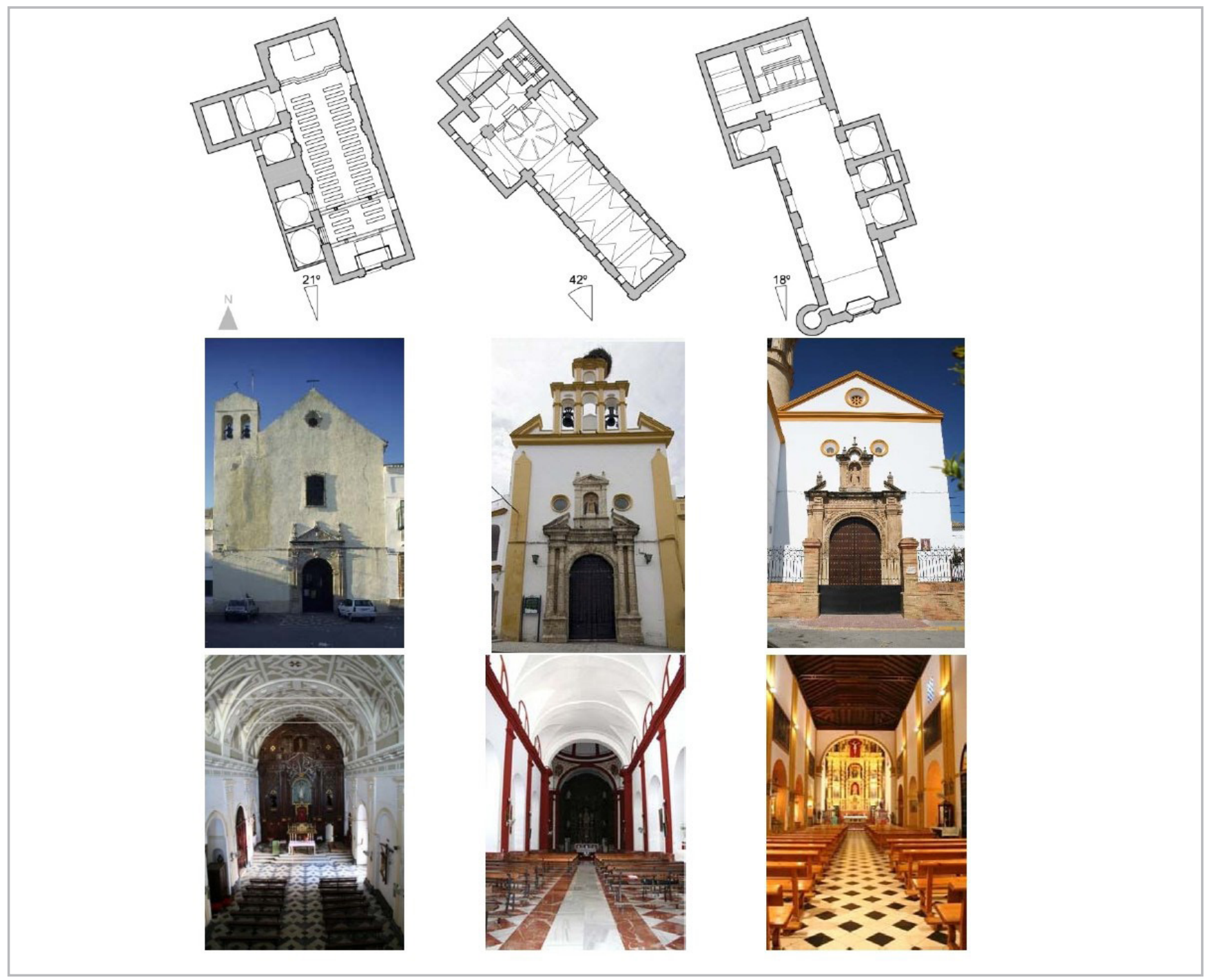

Figura 2.- Muestra de las iglesias de estudio. 1) Iglesia de San Francisco 2) Iglesia de Na Señora de la Merced 3) Iglesia de Na Señora de la Victoria.
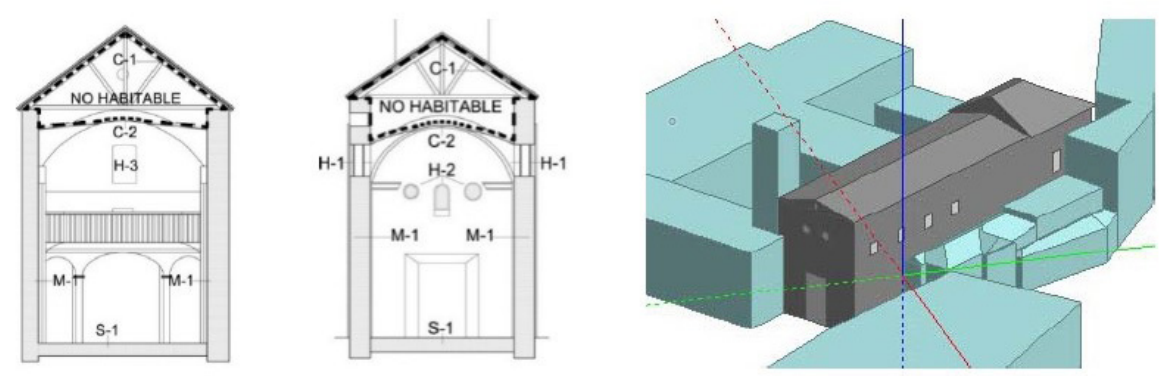

Figura 3.- Modelos tridimensionales. 1) Iglesia de San Francisco 2) Iglesia de Na Señora de la Merced 3) Iglesia de Na Señora de la Victoria.

Tabla 1.- Características constructivas de las iglesias

\begin{tabular}{|l|l|l|l|}
\hline \multicolumn{1}{|l|}{ San Francisco } & Ntr. ${ }^{\text {a. }}$ Señora de la Merced & Ntr. ${ }^{\text {. }}$ Señora de la Victoria \\
\hline Muros externos & Mampostería y ladrillos & Mampostería y ladrillos & Mampostería y ladrillos \\
\hline Bóveda & Piedra y madera & Piedra y madera & - \\
\hline Cubierta & Tejas árabes, madera y cañizos & Tejas árabes, madera y cañizos & Tejas árabes, vigas de acero, artesonado de madera \\
\hline Suelos & Mármol & Mármol & Mármol \\
\hline Ventanas & Vidrio simple & Vidrio doble & Vidrio simple \\
\hline
\end{tabular}




\section{Elaboración de modelos tridimensionales para la simulación del campo higrotérmico}

El interior de estos espacios religiosos fue monitorizado para realizar un seguimiento del ambiente interior para proporcionar así datos objetivos para realizar una evaluación de diagnosis del estado de conservación del patrimonio inmueble y mueble. Igualmente, uno de los avances en este estudio, fue que estos datos obtenidos de mediciones de temperatura del aire, temperatura superficial, humedad relativa y absoluta y velocidad de aire interior se utilizarían como referencia real para los procesos de validación de las simulaciones de modelos virtuales. Esto nos permitió, tener una herramienta fundamental dentro de la metodología aplicada a los criterios de diseño del acondicionamiento ambiental de edificios patrimoniales.

Los datos de monitorización se emplearon para generar los modelos de simulación informática, elaborando mo- delos digitales tridimensionales de cada iglesia de estudio. Se realizaron varios modelos espaciales que reproducían cada iglesia y su entorno, desde el punto de vista geométrico, climático y constructivo, empleando el programa informático Design Builder.

Los modelos informáticos tridimensionales tenían un comportamiento higrotérmico muy similar al que había en la realidad. La validación de un modelo consistió en la comparación de los resultados simulados con los datos reales recogidos en un período de tiempo. La metodología de validación se basó en la comparación de un importante número de datos horarios registrados in situ con los resultados obtenidos de la simulación informática una vez generado el modelo. Un modelo era válido cuando la variación de las mediciones in situ y las del modelo virtual, difería menos de $1^{\circ} \mathrm{C}$ de temperatura y $1.5 \mathrm{~g} / \mathrm{m}^{3}$ de humedad absoluta durante el $95 \%$ del tiempo y menos del $5 \%$ de humedad relativa durante el $90 \%$ del tiempo. Véase [figura 4]
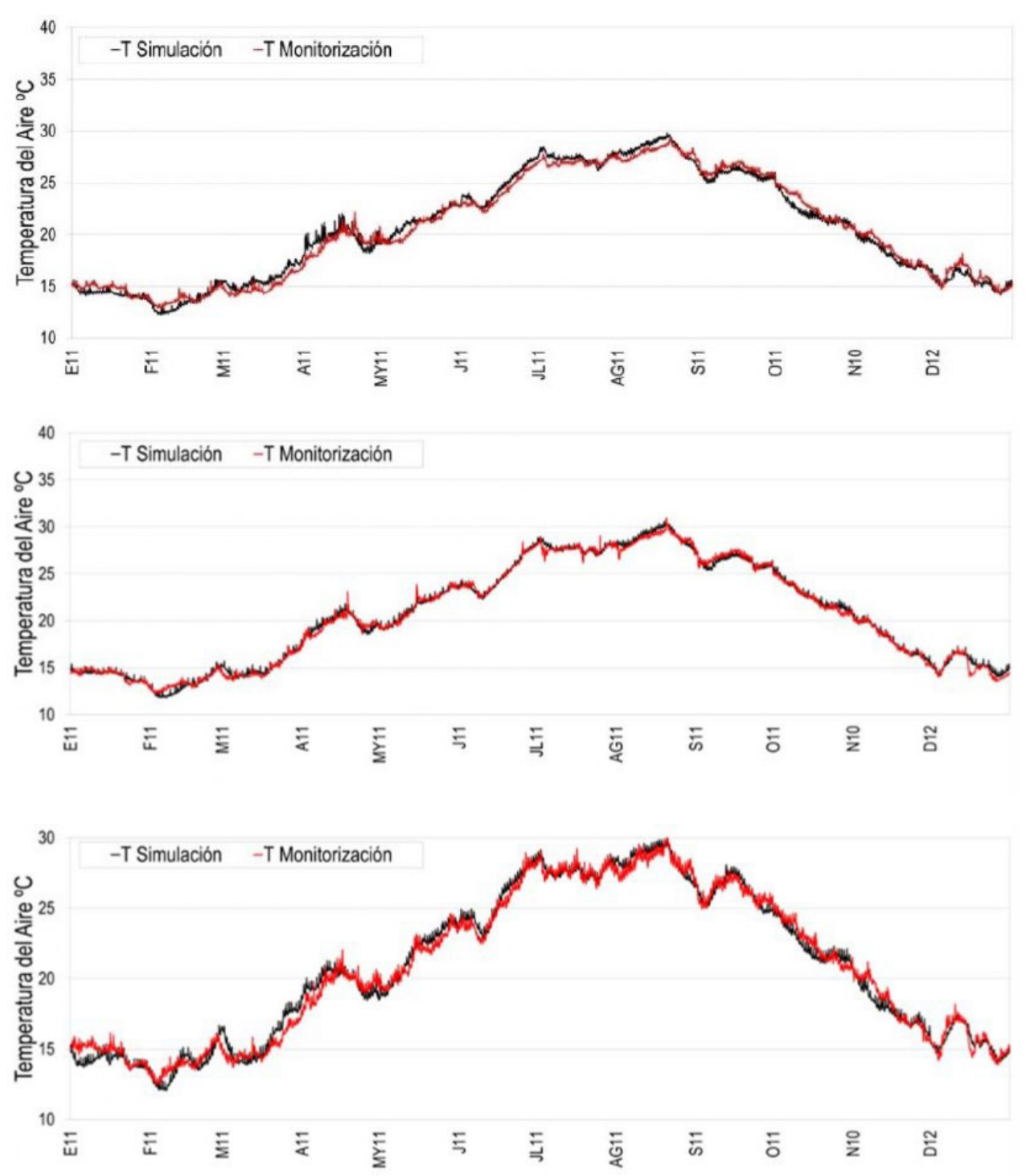

Figura 4.- Validación de modelo tridimensionales, temperatura del aire. 1) Iglesia de San Francisco 2) Iglesia de Na Señora de la Merced 3) Iglesia de Na Señora de la Victoria. 


\section{Resultados y discusión}

A continuación, se muestran los resultados y el análisis global del comportamiento higrotérmico de la muestra en el caso de la aplicación de las técnicas ambientales activas y mixtas.

-Monitorización y realización de mediciones in situ, procesado y valoración higrotérmica

Las medidas higrotérmicas llevadas a cabo in situ en cada una de las iglesias objeto de estudio, nos permitió evaluar y caracterizar su comportamiento higrotérmico. Estos edificios se caracterizaban por presentar una elevada inercia térmica, derivada de sus sistemas constructivos. Asimismo, al estar rodeadas de otras edificaciones, la incidencia e influencia solar sobre sus cerramientos exteriores era mínima.

Las mediciones reflejaron que las diferencias de temperatura y humedad relativa interiores (valores promedios) no eran significativos, excepto en los meses más cálidos (finales de primavera, verano y principios de otoño). Véase [figura 5].

En verano, o cuando las temperaturas interiores eran más elevadas, la humedad absoluta interior aumentaba sensiblemente por la evaporación del agua contenida en el suelo y parte inferior de muros (humedad de capilaridad).
Los meses más fríos y en primavera la temperatura interior tenía una mayor incidencia sobre la humedad absoluta.

Igualmente, se caracterizó la temperatura radiante interior de la muestra de iglesias mediante el empleo de equipos de medida infrarrojas. En los ensayos realizados se observó que en invierno se producía un ligero gradiente térmico vertical en los muros que era apreciable principalmente a últimas horas del día. Esa diferencia de temperatura entre las partes baja y alta se debía a la estratificación del aire y a la influencia de la radiación solar sobre las cubiertas [figura 6]. La diferencia de temperatura oscilaba entre 0.5 ${ }^{\circ} \mathrm{C}$. En verano, el gradiente térmico era más acusado entre las zonas altas y bajas de la iglesia, en torno a $2.5^{\circ} \mathrm{C}$.

Como era esperable, los únicos flujos de calor significativos de la envolvente se observaban en los elementos constructivos de menor resistencia e inercia térmica: huecos de muros y cubierta.

En todas las iglesias se detectaron humedades de capilaridad en la parte baja de los muros que provocaban evaporación de agua y aumento la de la humedad ambiental interior.

El suelo de las iglesias era la superficie que presentaba una temperatura radiante más baja (entre $21^{\circ} \mathrm{C}$ en verano y $14{ }^{\circ} \mathrm{C}$ en invierno). Esto generaba que, en verano, este elemento actuara como disipador de cargas térmicas y regulador de temperatura interior.

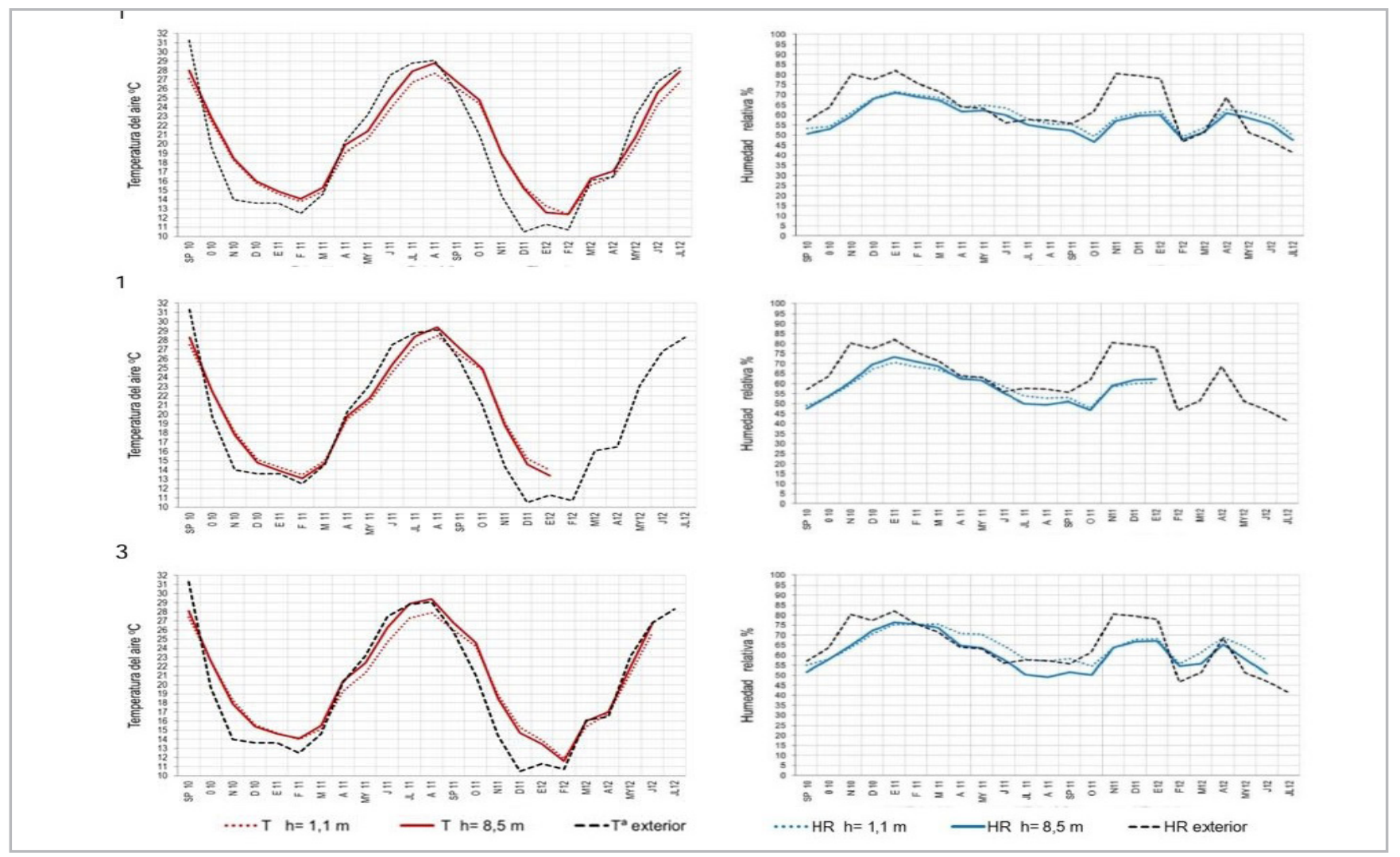

Figura 5.- Valores medidos de Ty HR (Promedios mensuales). 1) Iglesia de San Francisco 2) Iglesia de Na Señora de la Merced 3) Iglesia de Na Señora de la Victoria. 


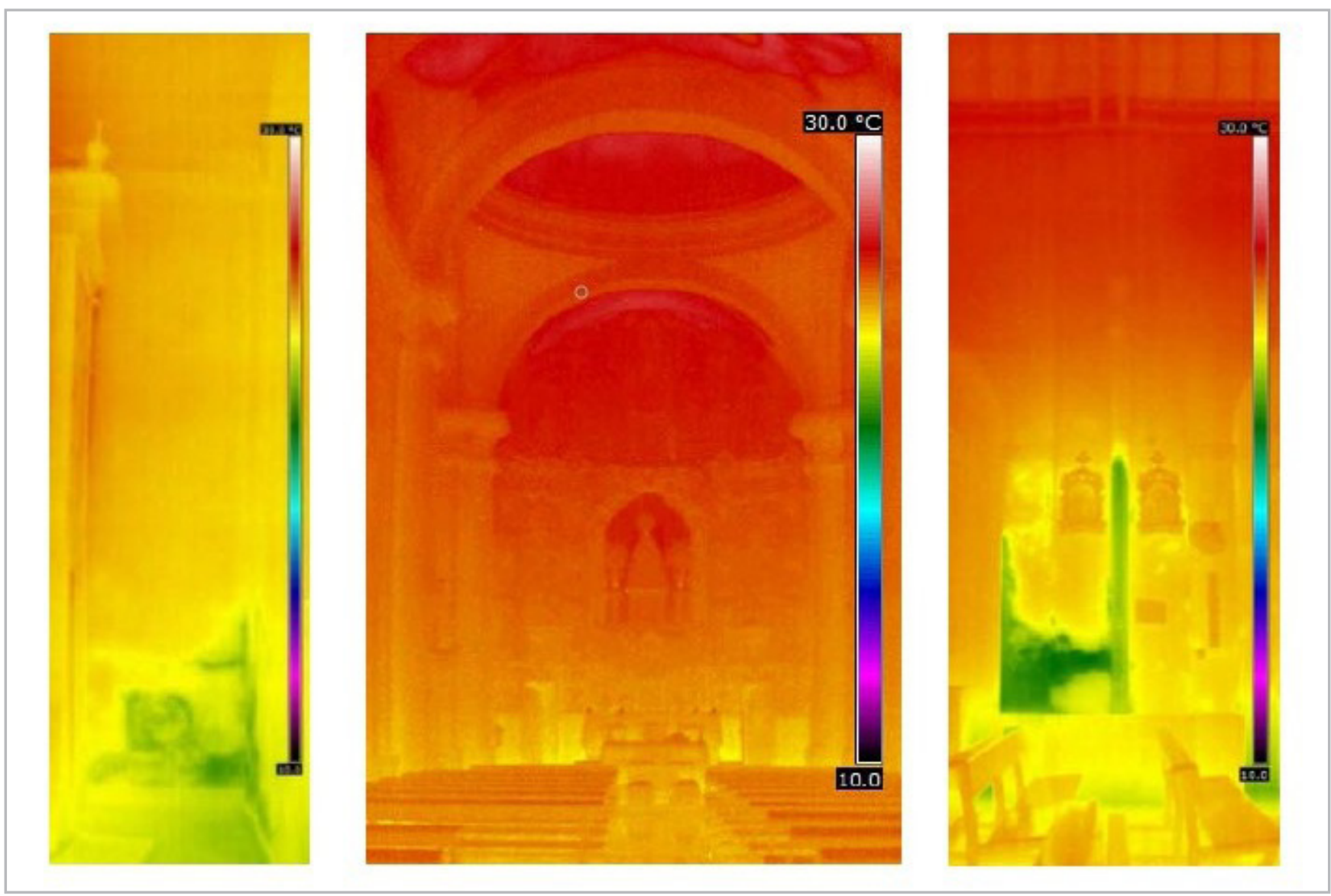

Figura 6.- Temperatura radiante en el mes de junio. 1) Iglesia de San Francisco 2) Iglesia de Na Señora de la Merced 3) Iglesia de Na Señora de la Victoria.

\begin{tabular}{|c|c|c|c|c|}
\hline \multicolumn{5}{|c|}{ IGLESIA SAN FRANCISCO DE ASÍS } \\
\hline & OTOÑO & INVIERNO & PRIMAVERA & VERANO \\
\hline Sin riesgo & $19 \%$ & $23 \%$ & $15 \%$ & $0 \%$ \\
\hline Riesgo bajo & $80 \%$ & $77 \%$ & $55 \%$ & $88 \%$ \\
\hline Riesgo alto & $1 \%$ & $0 \%$ & $30 \%$ & $12 \%$ \\
\hline \multicolumn{5}{|c|}{ IGLESIA NUESTRA SEÑORA DE LA MERCED } \\
\hline & OTOÑO & INVIERNO & PRIMAVERA & VERANO \\
\hline Sin riesgo & $24 \%$ & $25 \%$ & $20 \%$ & $0 \%$ \\
\hline Riesgo bajo & $74 \%$ & $75 \%$ & $73 \%$ & $98 \%$ \\
\hline Riesgo alto & $2 \%$ & $0 \%$ & $7 \%$ & $2 \%$ \\
\hline \multicolumn{5}{|c|}{ IGLESIA NUESTRA SEÑORA DE LA VICTORIA } \\
\hline & OTOÑO & INVIERNO & PRIMAVERA & VERANO \\
\hline Sin riesgo & $14 \%$ & $13 \%$ & $6 \%$ & $14 \%$ \\
\hline Riesgo bajo & $81 \%$ & $87 \%$ & $69 \%$ & $81 \%$ \\
\hline Riesgo alto & $5 \%$ & $0 \%$ & $25 \%$ & $5 \%$ \\
\hline
\end{tabular}

Tabla 2.- Porcentaje de tiempo en riesgo biodeterioro.
En cuanto al riesgo de biodeterioro del contenido mueble de las diferentes iglesias [tabla 2], el análisis de las mediciones in situ puso de manifiesto que durante los meses de primavera y verano era cuando tenía lugar los mayores periodos de riesgo al biodeterioro.

Igualmente, la pluviometría tenía una notable influencia en el aumento del riesgo al biodeterioro. En los años más lluviosos, el porcentaje de datos con riesgo alto al biodeterioro era mayor.

Del análisis del riesgo mecánico sobre el patrimonio mueble de las iglesias se concluyó que las desviaciones de la humedad relativa tenían una mayor incidencia sobre la preservación de las obras de arte, pues el porcentaje de tiempo en riesgo era mayor que en las desviaciones de temperatura. En general, las estaciones que presentan un mayor porcentaje de tiempo en riesgo, debido a las variaciones de la humedad relativa, eran otoño, invierno y primavera.

- Elaboración de modelos tridimensionales para la simulación del campo higrotérmico y ajuste de los modelos informáticos a partir de las mediciones in situ

Para validar los modelos de simulación higrotérmica en este tipo de edificios, fue básico fijar adecuadamente la 
temperatura del terreno, ya que tenía gran influencia en los resultados. A diferencia de otros investigadores, que proponen considerar los suelos en contacto con el terreno como elementos adiabáticos, en el caso que nos ocupa se comprobó que cuando incluíamos este como adiabático no se validaban los modelos de simulación, especialmente en verano, ya que este componente constructivo actuaba como disipador de calor (Huijbregts, Schellen, Schijndel \& Ankersmit 2015). Esto es una condicionante del clima, Mediterráneo, debido a las elevadas temperaturas en los meses de primavera y verano. Para poder validar los modelos de simulación, fue necesario medir in situ la temperatura superficial del suelo y fijar para el terreno una temperatura $2^{\circ} \mathrm{C}$ por debajo.

La humedad contenida en los materiales de construcción, que es bastante frecuente en estas construcciones, dificultaba la validación de los modelos, ya que alteraba las condiciones ambientales por el efecto de evaporación del agua en el interior del edificio. Por ello, se consideró necesario utilizar herramientas que permitieran simular este efecto. Así pues, en edificios históricos que presenten humedad en los muros por capilaridad, es necesario utilizar herramientas de simulación que permitan variar la humedad absoluta.

- Propuestas de mejoras del acondicionamiento ambiental de la muestra para la conservación y el confort térmico y simulación informática de las propuestas

Las diferentes propuestas de estudio se dividieron en varios bloques, técnicas ambientales pasivas: (conjunto de acciones de rehabilitación de la envolvente de la iglesia); técnicas ambientales activas (implementación de sistemas de climatización permanente).

En función a la evaluación de estas técnicas, las especificaciones ambientales para cada edificio tuvieron en consideración un amplio rango de materiales y sus combinaciones. La humedad relativa fue el parámetro más crítico en este campo y en consecuencia debía mantenerse en un nivel determinado tan estable como fuera posible. Al definir el límite del rango definitivo, se supuso las variaciones de HR para limitar daños mecánicos ocasionados por el microclima en materiales orgánicos higroscópicos de acuerdo con la Norma UNE-EN 15757 (UNE-EN 15757, 2011). Igualmente se fijaron los límites superiores para HR para evitar un biodeterioro por mohos, pudriciones, insectos, etc. Finalmente, según las normativas anteriores y la UNE-E-EN 15759-1 (UNE-E-EN 15759-1 2012) se establecieron los siguientes valores:

El modo de funcionamiento de los diferentes sistemas de climatización vino determinado principalmente por la necesidad de encontrar un equilibrio entre los requisitos para la conservación y el confort térmico, el tipo de utilización del edificio y la eficiencia energética. Los tipos de funcionamiento propuesto fueron los siguientes:

Funcionamiento 24 horas: El objetivo era proporcionar un microclima especificado de forma permanente, durante todo el año. El sistema de climatización funcionaba las 24 horas del día.

Funcionamiento 12 horas: Se proporcionaba un microclima especificado durante un periodo de tiempo limitado. En este caso el sistema funcionaba sólo durante el día, de 9.00 a.m. hasta 22.00 p.m.

Funcionamiento régimen de uso de la iglesia: Proporcionaba unas condiciones ambientales adecuadas únicamente cuando se utilizaba la iglesia, durante la celebración del culto. Debido a la inercia térmica de la iglesia, los sistemas debían encenderse al menos una hora antes de que comenzara la misa.

\section{-Análisis y valoración de las condiciones ambientales tras la implantación de técnicas ambientales}

Después de aplicar y combinar distintas técnicas ambientales pasivas y activas se observó que las propuestas de acondicionamiento ambiental que solían llevarse a cabo en los espacios religiosos situados en zonas climáticas más frías (norte y centro de Europa), no tenían la misma validez de aplicación en climas templados (clima Mediterráneo), debido a que las primeras estaban orientadas básicamente para condiciones extremas durante invierno.

Las distintas técnicas ambientales pasivas consideradas en esta investigación no garantizaban el confort a lo largo de un año, especialmente durante las estaciones de invierno y primavera. Las propuestas de rehabilitación que utilizaban exclusivamente técnicas ambientales pasivas no conseguían eliminar totalmente el riesgo mecánico y el riesgo al biodeterioro de los materiales que forman parte del patrimonio mueble.

Tabla 3.- Condiciones interiores de diseño para la conservación y el confort humano

\begin{tabular}{cccccccc}
\hline \multirow{2}{*}{ Condiciones típicas } & \multicolumn{3}{c}{ Temperatura operativa } & \multicolumn{2}{c}{ Velocidad } & Humedad \\
\cline { 2 - 5 } relativa \\
Invierno & mínima & óptima & máxima & mínima & óptima & máxima & $30-65 \%$ \\
Verano & 20 & 22 & 24 & 0,05 & 0,15 & 0,25 & $30-65 \%$ \\
\hline
\end{tabular}


Cuando se aplicaban simultáneamente varias técnicas pasivas se lograba paliar los efectos anteriores. La combinación que conseguía los mejores resultados para la preservación del patrimonio era la que contempla la utilización de doble ventana, aislamiento de las cubiertas (bóvedas o artesonados), aislamiento térmico de muros y suelo.

Las intervenciones que incorporaban aislamiento térmico en el suelo de las iglesias repercutían desfavorablemente en el confort térmico de los feligreses, ya que se eliminaba su efecto como disipador térmico durante gran parte del año.

La aplicación de técnicas ambientales activas, en combinación con las pasivas, en régimen de funcionando durante $24 \mathrm{~h}$ y $12 \mathrm{~h}$, mejoraba las condiciones ambientales originales y disminuía el riesgo de degradación, no existiendo datos propicios para que se produjera el biodeterioro de los de las obras de arte.

Los sistemas activos estudiados que no realizan un control absoluto de la humedad interior (humectación o deshumectación), como era el caso de equipo autónomo, UTA (Unidad de Tratamiento del Aire) a dos tubos y solo ventilación agravaban la situación inicial, ya que los límites establecidos para este parámetro se superaban en primavera, verano y otoño, existiendo, por tanto, riesgo de deterioro del patrimonio mueble.

Cuando los sistemas activos de acondicionamiento ambiental actuaban únicamente cuando el edificio estaba ocupado (régimen de uso), existía riesgo de biodeterioro principalmente en primavera, en torno al $5 \%$ del tiempo.

El riesgo de daño mecánico sobre el patrimonio mueble de la muestra, no se producía si los sistemas activos funcionaban durante las $24 \mathrm{~h}$, excepto en aquellos casos donde los equipos no controlan la HR, o cuya función exclusiva era la ventilación de las iglesias. Cuando el periodo de funcionamiento era de $12 \mathrm{~h}$, existía la posibilidad de que las obras sufrieran daños mecánicos durante el $10 \%-15 \%$ del tiempo y con el funcionamiento en régimen de uso durante el $5 \%-10 \%$ del tiempo.

\section{Conclusiones}

Los edificios religiosos son una parte importante del patrimonio cultural, son documentos de nuestra herencia y tenemos la necesidad de preservarlos para el presente y para las futuras generaciones.

Las características esenciales de estos edificios son sus sistemas constructivos (antiguas técnicas de edificación); que no fueron diseñados como viviendas o como espacios de trabajo con un uso discontinuo, y que por su decoración son edificios vulnerables.
Originalmente, la mayoría de los lugares históricos de culto no estaban acondicionados, pero actualmente, estos edificios están siendo climatizados para proporcionar el confort térmico a los fieles y visitantes y para mejorar las condiciones del clima interior para la conservación de los edificios y de su contenido o para lograr una combinación de ambas. No obstante, los requisitos normales de climatización para el confort térmico pueden estar en conflicto con los de la conservación, y por lo tanto se necesita llegar a un equilibrio.

Muchos de estos recintos están siendo equipados de sistemas de acondicionamiento ambiental $y$, en muchos casos, de forma inadecuada. Así pues, para tratar estas cuestiones debemos cambiar la manera de intervenir en estos edificios existentes, de modo que reduzcamos los impactos negativos en su preservación y en el medioambiente.

Así que, con el fin de hacer frente a estos retos, se desarrolló una metodología que identificara el modo más adecuado de acondicionar estos espacios. La metodología utilizada en este estudio pudo evaluar la aplicación de técnicas ambientales pasivas y activas en las iglesias históricas localizadas en un clima Mediterráneo.

Con este trabajo de investigación se ha procurado contribuir en el aporte de soluciones a un problema funcional que se está produciendo hoy en día y donde las técnicas que se están aplicando no están presentando, en general, resultados óptimos. La aplicación de un método experimental contrastado permitió afirmar que la implementación de las propuestas de rehabilitación ambiental mejoraría las condiciones ambientales para la preservación del patrimonio y el confort humano. Igualmente, se pudo determinar el consumo de la instalación y estudiar previamente la viabilidad del sistema.

El presente trabajo, ha propuesto profundizar en el conocimiento del acondicionamiento higrotérmico de espacios religiosos para ofrecer a los técnicos competentes las pautas de diseño que le permitan predecir la conducta higrotérmica de estos espacios antes y después de la aplicación de técnicas ambientales.

\section{Referencias}

BALOCCO, C., COLAIANNI, A. (2018)."Assessment of energy sustainable operations on a historical building. The Dante Alighieri High School in Florence". Sustainability, 10 (6), 2054: 1-24. https://doi.org/10.3390/su10062054

BERARDINIS, P., ROTILIO, M., CAPANNOLO, L. (2017). “Energy and Sustainable Strategies in the renovation of existing buildings: an Italian Case Study". Sustainability, 9 (8) 1472: 1-20. https://doi.org/10.3390/su9081472 
CAMUFFO, D., PAGAN, E., RISSANEN,S., BRATASZ, L., KOZLOWSKI, R., CAMUFFO, M. V. (2010). "An advanced church heating system favourable to artworks a contribution to European standardisation". Journal of Cultural Heritage, 11: 205-219. https://doi.org/10.1016/j.culher.2009.02.008

\section{DIRECCIÓN GENERAL DE BELLAS ARTES Y BIENES CULTURALES} Y DE ARCHIVO Y BIBLIOTECA. (2009). “Conservación preventiva en lugares de culto". Actas de las jornadas celebradas en el Instituto del Patrimonio Cultural de España. Madrid: Ministerio de Educación, Cultura y Deporte.

ERHARDT, D., MECKLENBURG, M. (1994). "Relative Humidity re-examined en Preventive conservation. Practice, Theory and Research". London: The contributions to the Ottawaz II Congress. Roy, Ashok and Smith, Perry: 32-38.

HUIJBREGTS, Z., SCHELLEN, H., SCHIJNDEL, J., ANKERSMIT, B. (2015). "Modelling of heat and moisture induced strain to assess the impact of present and historical indoor climate conditions on mechanical degradation of a wooden cabinet". Journal of Cultural Heritage, 16: 419-427. https://doi. org/10.1016/j.culher.2014.11.001

MAGRINI, A. Y. (2016). “The energy performance improvement of historic buildings and their environmental sustainability assessment". Journal Cultural Heritage. 21: 834-841. https:// doi.org/10.1016/j.culher.2016.03.012

MOSOARCA, M., et. al. (2017). "Failure analysis of historical buildings due to climate change". Engineering Failure analysis, 82:666-680.https://doi.org/10.1016/j.engfailanal.2017.06.013

MUÑOZ, C. (2016)."Air conditioning and passive environmental techniques in historic churches in Mediterranean climate. A proposed method to assess damage risk and thermal comfort pre-intervention, simulation". Energy and Buildings, 130: 567577. https://doi.org/10.1016/j.enbuild.2016.08.078

MUÑOZ-GONZALEZ, C. (2016). "Study of the implantation of the technologies of hygrothermal conditioning in the refurbishment project of churches. The case of Baroque churches in Moron de la Frontera". [PhD thesis]. Sevilla.

UNE-E-EN 15759-1.(2012). “Conservación del patrimonio cultural. Clima interior. Parte 1: Recomendaciones para la calefacción de iglesias, capillas y otros lugares de culto". Madrid.

UNE-EN 15757. (2011). “Conservación del patrimonio cultural. Especificaciones de temperatura y humedad relativa para limitar los daños mecánicos causados por el clima a los materiales orgánicos higroscópicos “. Madrid.

WESSBERGA, M., VYHLÍDAL, T. (2019). “A model-based method to control temperature and humidity in intermittently heated massive historic buildings". Sweden: Building and Environment, 159 (15): 1-16. https://doi.org/10.1016/j.buildenv.2019.03.024

\section{Autor/es}

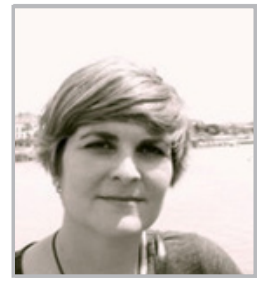

Carmen M.a Muñoz González

carmenmgonzalez@uma.es

Departamento de Construcciones

Arquitectónicas. Profesora Sustituta

Interina. Universidad de Málaga

En el año 2008 finalizó sus estudios en la E.T.S de Arquitectura. Compaginando con el trabajo como arquitecta en estudios de arquitectura comenzó el Máster de Arquitectura y Patrimonio Histórico en el año 2008. Ese mismo año se incorporó como colaboradora en el Departamento de Construcciones Arquitectónicas I de la E.T.S.A. de la Universidad de Sevilla. Actualmente trabaja como profesora en del Departamento de Construcciones Arquitectónicas del a Universidad de Málaga y forma parte del grupo de investigación denominado TEP130: Arquitectura, Patrimonio y sostenibilidad: Acústica, Iluminación, Óptica y Energía, perteneciente al Plan Andaluz de Investigación, Desarrollo e Innovación (PAIDI). Ha trabajado como investigadora en varios proyectos de investigación desde el año 2010 hasta la actualidad, TECNOCAI-ACCIONA, Caracterización arquitectónica del parque de ascensores y su huella ecológica, SUB-UMBRA y Rehabitar el Carmen, Plan de rehabilitación energética del patrimonio residencial malacitano. En el año 2012 comenzó su doctorado con mención internacional, el cual finalizó en 2016. Su trayectoria en el campo de investigación se ha centrado en la línea de Building, Environment \& Energy, y especialmente en dos líneas: Energy Efficiency and Lighting.

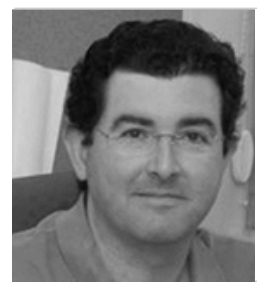

Ángel Luis Rodríguez León
leonr@us.es
Departamento de Construcciones
Arquitectónicas Profesor Titular
Universidad de Sevilla

Desde el año 1996, fecha en la que finalizo sus estudios en la E.T.S. de Arquitectura, forma parte del grupo de investigación denominado TEP130: Arquitectura, Patrimonio y Sostenibilidad: Acústica, Iluminación, Óptica y Energía, perteneciente al Plan Andaluz de Investigación, Desarrollo e Innovación. Mantiene una actividad docente e investigadora desde hace 22 años. Ha sido responsable de varias asignaturas de Acondicionamiento ambiental e Instalaciones del título de Arquitecto, siendo profesor en varios cursos de doctorado y títulos oficiales de máster, tanto de carácter nacional e internacional. En la actualidad es director del Título Propio de la US denominado Máster en Proyecto de Instalaciones en arquitectura: diseño, cálculo y eficiencia energética. Su trayectoria dentro del citado grupo de investigación se ha centrado en la línea: Building, Environment \& Energy, y específicamente en dos sub-líneas: Room Acoustics y Energy Efficiency. 


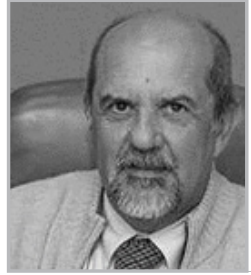

\section{Jaime Navarro Casas}

jnavarro@us.es

Departamento de Construcciones

Arquitectónicas

Catedrático

EsdoctorarquitectoyCatedráticodeConstrucciones Arquitectónicas desde 1984. Ha sido director del Instituto Universitario de Ciencias de la Construcción (I.U.A.C.C) de la Universidad de Sevilla, director de la Escuela Técnica Superior de Arquitectura de Sevilla y además de miembro de la Comisión de Investigación de dicha Universidad. Forma parte del grupo de investigación denominado TEP130: Arquitectura, Patrimonio y Sostenibilidad: Acústica, Iluminación, Óptica y Energía, perteneciente al Plan Andaluz de Investigación. Actualmente, es el arquitecto de la Catedral de Sevilla. Es miembro de la comisión Docente del Máster Arquitectura y Restauración del Patrimonio Histórico. Su trayectoria dentro del citado grupo de investigación se ha centrado en la línea: Building, Environment \& Energy, y específicamente en dos sub-líneas: Room Acoustics y Energy Efficiency and Artificial and Natural Lighting.

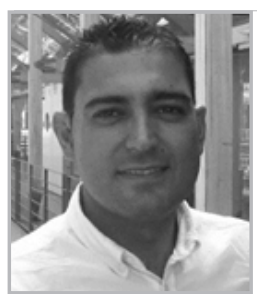

\section{Jonathan Ruiz Jaramillo}

jonaruizjara@uma.es

Departamento de Construcciones

Arquitectónicas Profesor Ayudante Doctor

Universidad de Málaga

Arquitecto por la Universidad de Sevilla y Master en peritación y reparación de edificios. Doctor Arquitecto desde 2012, su tesis doctoral titulada "Comportamiento sísmico de edificios históricos. Las iglesias mudéjares de Sevilla" obtiene el premio Focus-Abengoa en el año 2013. Su labor investigadora se inicia en el año 2006 obteniendo una beca de formación de personal investigador (FPI) en el Departamento de Construcciones Arquitectónicas en la E.T.S. de Arquitectura de Sevilla en el marco del proyecto de investigación "Obtención de un método que garantice y optimice el empleo de elementos de madera al exterior frente a los agentes medioambientales". En el año 2011 se incorpora como profesor del Área de Construcciones Arquitectónicas de la ETS de Arquitectura de la Universidad de Málaga, donde en la actualidad desarrolla su actividad docente e investigadora. Ha participado en diversos proyectos de investigación como "Comportamiento sísmico de edificios construidos con técnicas tradicionales (fábrica de piedra, ladrillo, adobe, tapial, madera, etc.). Evaluación de la seguridad estructural. Propuesta de técnicas de rehabilitación no destructivas"; "Obtención de un método no contaminante y sustentable que permita la conservación de edificios antiguos construidos en madera. Aplicación al centro histórico de Morelia (México)"; "Restauración de bienes muebles e inmuebles en madera"; "Caracterización de materiales, componentes y estimación de características resistentes de iglesias altiplánicas (adobe y quincha), museo del olivo en quinto (tapial), restos tartésicos del carambolo"; "Norma sísmica. Análisis previo y rehabilitación ante daños de edificios e infraestructuras existentes". En la actualidad, junto a Luis García Pulido, es investigador principal del proyecto "Las atalayas que defendieron el reino nazarí de Granada. Análisis y documentación científica (NAZALAYA)”. .

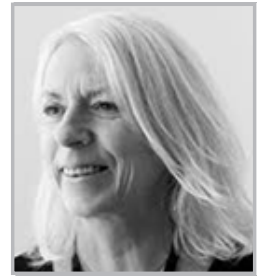

Catherine Teeling

catherine.teeling@port.ac.uk Department Portsmouth School of Architecture. Lecture.Creative and Cultural Industries. Universidad de Portsmouth

After a career in practice of over 12 years and following the completion of a masters degree in 'Computation in Design' in 1996, Catherine has been working in academia as a senior lecturer in Architecture, firstly at the University of Greenwich teaching post grad level Architecture \& as course leader for Masters in Urban Design. Where she secured 249K funding from HEFCE (Higher Education Funding Council of England) for a three year best practice learning and teaching project exploring the 'Creative use of Computers in Design' and established the first Electronic Design Studio (EDS) within a School of Architecture in the UK. The culmination of the project resulted in the first International Symposium on Digital Creativity in 2000. Catherine has worked at UCLA and undertook a six-month sabbatical as a visiting professor at Massachusetts Institute of Technology (MIT) in Boston, USA. Where she worked within the Computation Research Group and taught in the School of Architecture MIT. From 2003 she has been a Principal Lecturer and Course Director for masters in Architecture and undergraduate Interior Architecture \& Design, at the Canterbury School of Architecture now the University of the Creative Arts.

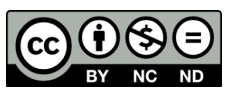

https://doi.org/10.37558/gec.v17i1.758 